\title{
Review of: "Clinical Features And Risk Factors For Infection In Patients With Rheumatoid Arthritis"
}

\author{
Elio Castagnola
}

Potential competing interests: The author(s) declared that no potential competing interests exist.

The paper is of interest but some very important data are lacking and there are not acceptable definitions. 1. data: it should be of great interest to know the duration of follow up of patients in order to define a rate of infections according to the duration of period at risk. Since AR requires long-term therapies it should be of utmost importance to know the frequency of infections during therapy. For example if $50 \%$ of patients develop an infection it is a very high proportion, but if this occurs during a 10 years follow up, the importance changes. Moreover it should be of interest also to know when the 1st episode occurred after the start of treatment (cumulative risk)

2. definitions of infections: to find Candida in the respiratory tract is of no interest since candida pneumonia does not exist, unless for hematogenous spread. Similarly, it should be important to know if S.aureus (ad all other pathogens) in respiratory tract was associated with a clinical picture of pneumonia or it represented only colonization. Of course these definitions will change dramatically the proportions of infectious complications. antibiotic susceptibility:

3.it should be state the system adopted to define that (CLSI, EUCAST, other?), today is not enough to report susceptible or resistant without the reference "frame"

In my opinion to give an aswer to these point is mandatory for a correct discussion of the results, that could be completely different from those presente here 